A.G. Mamalis, A.I. Grabchenko, V.A. Fedorovich, J. Kundrak, Y. Babenko and T. Dovbiy

\title{
Chemical deposition of nickel with inclusion of ultradispersed diamonds
}

ABSTRACT. In this paper the state-of-the-art technology of deposition of diamond-nickel coatings on synthetic diamond surfaces is described. Owing to the presence of nanodiamond particles, the coating is extremely hard and can be effectively used when manufacturing superabrasive tools.

Keywords: coating, diamond wheel, grinding, nanodiamond, ultradispersed diamond

Nanotechnology Perceptions 7 (2011) 218-222

Nonsubscribers purchase individual article 